\title{
Optical coherence tomography angiography versus fluorescein or indocyanine green angiography: legal implications for ophthalmologists
}

\author{
Gianfranco Gioia* and Mauro Salducci \\ Department of Sense Organs, Master of Medical Legal Ophthalmology (Director: Professor Mauro Salducci), Sapienza University of Rome, Rome, Italy
}

\begin{abstract}
Optical coherence tomography angiography (OCTA) is a non-invasive imaging technique able to show real-time volumetric data on chorioretinal vasculature. The most commonly used angiographic techniques in visualizing vascular changes are fluorescein (FA) or indocyanine green angiography (ICGA). Dye-based techniques require intravenous dye injection which can cause side effects. The physician has the responsibility to decide which diagnostic instrument is more appropriate. Legal implications are due to the side effects of dye injection. OCTA test can be performed without side effects.
\end{abstract}

\section{Introduction}

Optical coherence tomography angiography (OCTA) is a noninvasive imaging technique able to generate real-time volumetric data on chorioretinal vasculature with its flow pattern. The images of OCTA resemble an angiogram thanks to the efficient algorithms of high-speed optical coherence tomography and established in face chorioretinal segmentation. The principle of OCTA is based on the change in backscattering between consecutive B-scans thus attributing the differences to the erythrocytes flow through retinal blood vessels. OCTA is useful in the evaluation of several ophthalmologic diseases such as age-related macular degeneration, retinal vascular occlusions, and diabetic retinopathy [1]. OCTA has several important clinical findings such as areas of macular telangiectasia, impaired perfusion, microaneurysms, capillary remodelling, intraretinal fluid, and neovascularization. Its limitations include a relatively small field of view, inability to show leakage, and image artifacts. Further larger studies are mandatory to define OCTA utility in clinical settings to guide therapeutic interventions in retinal diseases [2].

The most commonly used angiographic techniques in visualizing vascular changes are fluorescein (FA) or indocyanine green angiography (ICGA). Fluorescein is useful to visualize the retinal vasculature, while ICGA is useful to show the choroidal vessels. Dye leakage and staining blur the boundaries of capillary dropout or neovascularization. These techniques show little depth information due to the two-dimensional nature of the images [3]. OCTA showed high sensitivity and specificity for detection of choroidal neovascularization. Concordance between OCTA and dye-based techniques was excellent. OCTA may represent a first-line non-invasive method for the diagnosis of neovascular agerelated macular degeneration [4].

Dye-based techniques require intravenous dye injection, which is time consuming and can cause side effects. The patient is informed about risks, but the physician has the responsibility to decide which diagnostic instrument is more appropriate considering for side effects, clinical conditions of the patients and the needed clinical data. Therefore, legal implications are due to the side effects of dye injection.
In a prospective study of 2789 angiographic procedures in 2025 patients, the percentage of adverse reactions depended strongly on the patient's angiographic history. Adverse reactions followed $4.8 \%$ of the angiographic procedures and included nausea (2.9\%), vomiting $(1.2 \%)$, flushing/itching/hives (0.5\%), and other reactions (dyspnea, syncope, excessive sneezing) (0.2\%). No cases of anaphylaxis, myocardial infarction, pulmonary oedema, or seizures occurred. The percentage of reactions was $48.6 \%$ for patients who had had an adverse reaction to angiography previously [5]. A Japanese study evaluated the safety of indocyanine green for use in fundus angiography. Ophthalmologists at 15 institutions reported a total of 3774 indocyanine green angiograms. The dosage of indocyanine green used varied from 25 to $75 \mathrm{mg}$. There were 13 cases of side effects (0.34\%), ten of which were mild reactions such as nausea, urtication, exanthema, itchiness, and urgency to defecate, one case of pain of the vein, which required treatment, and 2 cases of hypotension that required treatment for shock. The frequency of adverse reactions to indocyanine green and the frequency of such reactions to fluorescein sodium indicated that indocyanine green is safe as fluorescein in angiography [6].

An OCTA test can be performed quicker, without side effects and with more accuracy than FA or ICGA. OCTA provides us faster and more accurate images and information to assess vascular abnormalities and retinal diseases. So, the clinician has to choose the safest exam for the patients; its safety also allows OCTA to be performed more often than FA or ICGA in order to avoid legal implications consequent to side effects [7-9].

${ }^{*}$ Correspondence to: Gianfranco Gioia, Department of Sense Organs, Sapienza University of Rome, Viale del Policlinico,155, 00161, Rome, Italy, E-mail: gioiag86@hotmail.it

Key words: optical coherence tomography angiography, legal implications, dyebased techniques

Received: April 12, 2019; Accepted: April 24, 2019; Published: April 26, 2019 


\section{References}

1. Spaide RF, Fujimoto JG, Waheed NK, Sadda SD, Staurenghi G (2018) Optical coherence tomography angiography. Prog Retin Eye Res 64: 1-55. [Crossref]

2. Kashani AH, Chen CL, Gahm JK, Zheng F, Richter GM, et al. (2017) Optical Coherence Tomography Angiography: A Comprehensive Review of Current Methods and Clinical Applications. Prog Retin Eye Res 60: 66-100. [Crossref]

3. Cunha-Vaz J, Koh A (eds). (2018) Imaging Techniques. ESASO Course Series. Basel, Karger, Vol 10, pp 1-18.

4. Nikolopoulou E, Lorusso M, Micelli-Ferrari L, Cicinelli MV, Bandello F, et al. (2018) Optical Coherence Tomography Angiography versus Dye Angiography in Age-Related Macular Degeneration: Sensitivity and Specificity Analysis. Biomed Res Int 2018: 6724818. [Crossref]
5. Kwiterovich KA, Maguire MG, Murphy RP, Schachat AP, Bressler NM, et al. (1991) Frequency of adverse systemic reactions after fluorescein angiography. Results of a prospective study. Ophthalmology 98: 1139-1142. [Crossref]

6. Obana A, Miki T, Hayashi K, Takeda M, Kawamura A, et al. (1994) Survey of complications of indocyanine green angiography in Japan. Am J Ophthalmol 118: 749753. [Crossref]

7. Salducci M (2015) Validità dell'indagine clinica O.C.T. versus F.A.G. retinica nella diagnostica medico legale. Giornale di Medicina Militare. Rome Italy, Vol 1, pp: 20-32.

8. Salducci M, Pacella F, Collini S, Turchetti P, Smaldone G, et al. (2018) e Pacella E. Ropivacaine vs tetracaine in topical anesthesia for intravitreal injection. Sense Sci 2 . $106-110$

9. Salducci M (2018) Considerazioni medico legali sulla normativa attualmente vigente in Italia per il diritto all'indennità di accompagnamento con particolare riferimento agli ipovedenti. Prevent Res Scientific 2: 1.

Copyright: (02019 Gioia G. This is an open-access article distributed under the terms of the Creative Commons Attribution License, which permits unrestricted use, distribution, and reproduction in any medium, provided the original author and source are credited. 